Nouvelles perspectives en sciences sociales

Revue internationale de systémique complexe et d'études relationnelles

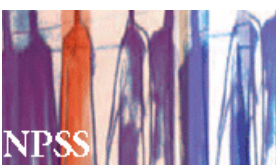

\title{
Dialectique de l’homogénéité et de la différence
}

\section{Simon Laflamme}

Volume 8, numéro 1, novembre 2012

URI : https://id.erudit.org/iderudit/1013916ar

DOI : https://doi.org/10.7202/1013916ar

Aller au sommaire du numéro

\section{Éditeur(s)}

Prise de parole

\section{ISSN}

1712-8307 (imprimé)

1918-7475 (numérique)

Découvrir la revue

\section{Citer cet article}

Laflamme, S. (2012). Dialectique de l'homogénéité et de la différence. Nouvelles perspectives en sciences sociales, 8(1), 15-33. https://doi.org/10.7202/1013916ar
Résumé de l'article

Deux grandes visions s'affrontent au $\mathrm{XX}^{\mathrm{e}}$ siècle : l'une veut que les sociétés humaines évoluent en se diversifiant, l'autre maintient que ces sociétés tendent vers la similitude. Mais ces visions sont nourries beaucoup plus par l'observation sommaire et l'autoréflexion que par l'analyse empirique. Les travaux empiriques qui se penchent sur cette problématique inclinent à relever une évolution double. Dans cet article, nous faisons état du positionnement contradictoire des visions en sciences humaines, nous tentons de fournir une explication de la double tendance que mettent en relief les travaux empiriques en en dialectisant les éléments et nous reprenons quelques-uns de nos travaux qui témoignent par l'observation de cette dialectique. 


\title{
Dialectique de l'homogénéité et de la différence
}

Simon Laflamme

Université Laurentienne

\begin{abstract}
Notre collaboration à ce numéro de la revue est pour nous I l'occasion de faire un bilan sur une thématique qui a animé notre réflexion à plusieurs reprises au cours des dernières années. Notre intention est, en premier lieu, de situer le questionnement sur l'homogénéité et la différence afin d'en rappeler à grands traits l'évolution et d'en souligner les particularités à compter du milieu du XXe. Elle est ensuite de revenir sur certains de nos travaux dans lesquels le questionnement a donné lieu à une opérationnalisation et, enfin, de dégager, grâce à cette évocation, quelques principes analytiques.
\end{abstract}

\section{Deux visions}

Dans la seconde moitié du $\mathrm{XX}^{\mathrm{e}}$ siècle, en Occident, deux visions antinomiques ont tenté d'expliquer le devenir de l'humanité, l'une insistant sur les phénomènes d'homogénéisation, l'autre sur ceux de différenciation. Elles sont encore vigoureuses toutes les deux. 


\subsection{Avant la révolution industrielle}

La préoccupation ne date pas de la fin du deuxième millénaire. Les présocratiques, déjà, se questionnaient sur la similitude et la dissimilitude, les Héraclite et les Empédocle mettant l'accent sur celle-ci, les Mélissos sur celle-là, les Anaxagore et les Démocrite les abordant toutes deux ${ }^{1}$. Mais la réflexion n'avait pas alors cours dans une perspective historique, l'histoire étant surtout perçue comme ce par quoi peuvent être pérennisés les noms et les événements; la réflexion ne portait pas directement sur ces phénomènes et elle avait davantage pour objet l'être que la socialité. Chez Héraclite, la diversité se révélait à travers les notions de contradiction et de mouvement; chez Empédocle, elle apparaissait dans une physique à quatre éléments (feu, air, terre et eau) dans laquelle agissent les forces de l'amour et de la haine. Chez Mélissos, c'est d'une philosophie de l'être, conçu comme immutabilité et unité que se dégage le semblable. Chez Anaxagore, une théorie de l'atome faisait que tous les corps sont fondamentalement pareils; elle cohabitait avec une interprétation du nôेs, une énergie qui assurait la spécificité des êtres. Chez Démocrite, une théorie de l'atome, à nouveau, ouvrait sur un univers dont les compositions étaient parfois semblables, parfois différentes, où la différence s'expliquait en fonction de la forme, de l'ordre et de la position.

La philosophie des Platon et des Aristote reprendra les questionnements des présocratiques et récupérera, développera, affinera les raisonnements. Tout au long du moyen-âge, la pensée grecque perdurera et les Saint Augustin puis les Saint Thomas d'Aquin veilleront à l'émailler de théologie chrétienne. On trouvera continûment les traces d'une curiosité pour l'homogénéité et l'hétérogénéité, mais toujours dans le cadre d'un discours qui se consacre à l'être.

$\overline{1}$ Roger Gervais, Presse et mondialisation : étude comparée franco-canadienne, thèse de doctorat, Université des sciences sociales de Toulouse I, Toulouse, mai 2009, p. 6-7. 


\subsection{Les premiers éléments philosophiques}

La révolution industrielle modifiera cette perspective. Avec elle, l'histoire sera moins étroitement perçue comme ligne de mémoire; la notion d'histoire sera associée à celle de transformation. La révolution industrielle aura imposé une analyse des changements qui se rapportent à la vie humaine, plus qu'à l'être. Autour des Lumières, la notion d'évolution se cristallisera; elle traduira une vision dans laquelle l'humanité suit un mouvement civilisateur. Les ouvrages des Karl Marx, Émile Durkheim et Max Weber, entre autres, ceux de la sociologie naissante, du milieu du XIX ${ }^{e}$ siècle jusqu'au début du XXe, seront largement des exposés sur les bouleversements de l'industrialisation dans lesquels l'humain apparaitra comme essentiellement social et historique.

Aux yeux de Marx, l'humanité, tout entière, suit un cours qui va des sociétés esclavagistes, aux féodales et aux capitalistes; viendront les sociétés socialistes puis le communisme. Les sociétés capitalistes sont des sociétés du même. Elles ne comptent réellement que deux ensembles : les bourgeois et les prolétaires. Les autres constituants ou bien sont appelés à prendre des formes de la société capitaliste ou de l'industrialisation, c'est le cas de la paysannerie, ou bien ne se définissent que par relation à la structure capitaliste, on peut songer au lumpen-prolétariat ou à la petite bourgeoisie. Les forces du capitalisme uniformisent les positions de classe en même temps que les modes de travail et les consciences $^{2}$. Durkheim note aussi une mutation des sociétés humaines, mais sa perspective n'est pas celle de Marx. Selon lui, auparavant, les sociétés comptaient peu de membres et elles étaient composées d'individus semblables les uns aux autres. Cette exiguïté et cette similitude rendaient possible une solidarité mécanique. Les sociétés industrialisées, elles, sont populeuses et leurs citoyens ont des caractéristiques variées. Cette étendue et cette hétérogénéité menacent l'harmonie du tout, de sorte que

$\overline{2}$ Karl Marx, Le Capital, tome 1, Presses universitaires de France, [1867] 1970; Karl Marx, Introduction à la critique de l'économie politique, Montreuil, Altiplano, [1857] 2008; Karl Marx et Friedrich Engels, Manifeste du parti communiste, Anjou (Québec), Éditions CEC, [1847] 2009. 
des institutions doivent veiller à transmettre quelques normes qui assureront une solidarité, laquelle sera organique, et non plus mécanique $^{3}$. L’optique wébérienne, sur ce point, est assez contrastée par rapport à de celles de Marx et de Durkheim. Il y a bien chez Weber une analyse de la modernité, la constatation d'une inadéquation entre une société industrialisée et une autre qui ne l'est pas. Mais il n'y a pas de théorie de l'histoire, comme chez Marx, ou de typologie diachronique des sociétés dont le critère est celui de la similitude, comme chez Durkheim. Weber propose une sociologie des individus qui ouvre d'emblée sur la diversité des actions. Mais il limite cet univers pluriel par une typologie de l'autorité qui canalise les activités; il le restreint aussi dans des analyses d'idéologies, notamment religieuses, qui collectivisent l'action humaine. La modernité, par ailleurs, se présente à lui comme un vaste processus de rationalisation qui altère le collectivisme qui découle des idéologies religieuses ${ }^{4}$.

\section{3. À compter du milieu du $\mathrm{XX}^{\mathrm{e}}$ siècle}

La première des deux visions qui, à compter du milieu du $\mathrm{XX}^{\mathrm{e}}$ siècle, imagineront le destin de l'humanité, s'alimentera essentiellement au marxisme. Elle dessinera à main levée un sombre tableau caractérisé par l'uniformité. C'est d'abord à l'École de Frankfort, notamment à Max Horkheimer et à Theodor W. Adorno, qu'on devra cette œuvré. L'image est simple. Le monde est divisé en deux : les capitalistes et les autres. Les capitalistes, propriétaires des moyens de production, sont privilégiés. Leur intérêt est de maintenir leur position. À cette fin, il leur faut

3 Émile Durkheim, De la division du travail social, Paris, Presses universitaires de France, $8^{e}$ édition, [1893] 1967; Émile Durkheim, Les Formes élémentaires de la vie religieuse, Paris, CNRS Éditions, [1912] 2007.

4 Max Weber : Économie et société, tome 1, Paris, Plon, [1921 (posthume)] 1971; Économie et société dans l'antiquité, Paris, La Découverte, [1909] 1998; Hindouisme et bouddhisme, Paris, Flammarion, [1916] 2003.

5 Max Horkheimer, Éclipse de la raison, suivi de Raison et conservation de soi, Paris, Payot, [1949] 1974. Theodor W. Adorno, Modèles critiques, tome 1 [1963], tome 2 [1965], Paris, Payot, 2003. Max Horkheimer et Theodor W. Adorno, La Dialectique de la raison : fragments philosophiques, Paris, Gallimard, coll. « Tel », [1947] 1983. 
aliéner les non-capitalistes pour empêcher qu'ils ne se révoltent, il leur faut donner préséance à l'économie marchande par rapport à toute autre considération. Le capitalisme, ainsi, homogénéise les esprits et les structures sociales à l'intérieur desquelles il opère. En modelant tout en marchandise, la culture elle-même devient objet de consommation, à des fins de profit. Le rapport à la culture se présente comme consommation des produits culturels. La jouissance l'emporte alors sur l'appréciation esthétique et sur la critique. L'art se dévoie. L'humain, dans ce processus, perd sa " raison ». Une bonne partie des sciences de la communication notamment celle qui développera la théorie des industries culturelles - redessinera le tableau désolant en mettant à l'avantplan les croquis de la marchandisation de la culture et ceux des impératifs d'aliénation. La figure capitaliste se révélera nettement dans sa dimension de propriétaire de moyens de production de messages culturels et les couleurs des messages mettront en évidence le projet de reproduction de l'ordre capitaliste. Dans une telle perspective, l'humanité ne peut se dévoiler autrement que comme entraînée vers la similitude ${ }^{6}$. Et c'est bien ainsi que de nombreux spécialistes des sciences sociales se représenteront son avenir ${ }^{7}$. Qu'on songe à ces auteurs de premier plan qui apercevront un processus d'homogénéisation dans les effets du marketing, comme Naomi Klein ${ }^{8}$, dans ceux de la production

$6 \quad$ Voir, par exemple : Jean Baudrillard, La Société de consommation : ses mythes, ses structures, Paris, Denoël, coll. "Folio Essai ", [1970] 1986; George Gerbner, "Mass Media and Human Communication ", dans Frank E. X. Dance (dir.), Human Communication Theory, New York, Holt, Rinehart, 1967, p. 40-57; Daniel Lerner, The Passing of Traditional Society, New York, Free Press, 1958; Armand Mattelart, Multinationales et système de communication, Paris, Anthropos, 1976; Bernard Miège, La Société conquise par la communication, Grenoble, Presses de l'Université de Grenoble, coll. «Logiques sociales ", 1996.

7 On lira avec intérêt, sur cette thématique, le chapitre I de la thèse de Roger Gervais, Presse et mondialisation : étude comparée franco-canadienne, op. cit.

8 Naomi Klein, No Logo. La tyrannie des marques, Arles, Actes Sud, [2000] 2001. 
des messages médiatiques, comme Pierre Bourdieu ${ }^{9}$ et dans ceux de la mondialisation, comme Ulrich Beck ${ }^{10}$.

La seconde vision se manifestera un peu plus tard dans le siècle. Elle s'inspirera du libéralisme, de la phénoménologie et même, parfois, du marxisme. Les portraits qu'elle dessinera mettront en relief l'individu. Elle en soulignera la subjectivité, l'aptitude à s'autodéterminer, à agir intentionnellement. Elle représentera cet acteur dans des scènes où il fait des choix. La société ne lui apparaîtra plus comme une entité dans laquelle les individus sont socialisés à des idéaux communs transmis par des institutions transcendantes. Les messages médiatiques, dans son regard, ne seront plus le produit d'une machine qui influe pareillement sur tous les esprits. Ces schémas, soutiendra-t-elle, appartiennent à la modernité; dans la postmodernité, les zones d'influence sont multiples. Une même personne est exposée à de nombreuses opinions par le fait qu'elle soit en lien avec des individus différents, les institutions qui agissent sur elle et dans lesquelles elle agit sont nombreuses et variables. L'école, par exemple, n'est plus un organisme dont les enseignants sont la réplique les uns des autres relayant des contenus identiques et les contenus varient selon les orientations que prennent les étudiants. Les parents ne transmettent pas le même discours indépendamment de la famille à laquelle ils appartiennent; les structures familiales sont nombreuses et peuvent même se transformer au cours de la vie d'un enfant. Un même individu est exposé à une grande diversité de messages médiatiques. L'individu est ainsi un carrefour particulier d'informations, plus ou moins intégrées. La postmodernité produit des individus; elle est un processus d'individuation ${ }^{11}$. L'individu lui-même se fragmente, détaché qu'il est

9 Pierre Bourdieu, Sur la télévision. L'emprise du journalisme, Paris, Raison d'agir, 1996.

10 Ulrich Beck, Pouvoir et contre pouvoir à l'ère de la mondialisation, Paris, Flammarion, [2002] 2003.

11 Yves Boisvert, Le Postmodernisme, Montréal, Boréal, 1995. Claude Dubar, La Crise des identités. Linterprétation d'une mutation, Paris, Presses universitaires de France, 2000. Anthony Giddens, Les Conséquences de la modernité, Paris, L'Harmattan, [1991] 2000; Antony Giddens, La Transformation de l'intimité. Sexualité, amour et érotisme dans les sociétés modernes, Rodez, Éditions du 
de sa communauté, incapable qu'il est de synthétiser les informations qui viennent à lui, de se réaliser à sa satisfaction ${ }^{12}$.

Ainsi, les sciences humaines livrent deux perspectives qui se contredisent : l'une qui annonce une humanité définie par la similitude, l'autre pour laquelle l'avenir humain est celui de la dissemblance.

Toutes les sciences humaines ne sont pas le fait de cette polarisation, bien sûr. Toutes ne baignent pas dans cet univers idéologique. En marge de ces positions macrologiques, on trouve plusieurs théories dans lesquelles on voit les structures produire de la similitude sans que cela empêche les particularismes. C'est le cas des théories de la socialisation dans lesquelles on montre que la société transmet des valeurs et des coutumes aux individus qui vivent en elle bien que ce processus n'empêche pas des intériorisations et des mises en ouvre différenciées ${ }^{13}$. C'est le cas aussi de la psychologie sociale qui, depuis Mead, associe l'individuel au collectif; Mead, en effet, soutient, par exemple, que la langue, extérieure qu'elle est aux individus, leur donne quelque chose en commun en même temps qu'elle rend possibles des expressions individuelles; l'individualité elle-même, aux yeux de Mead, suppose la socialité ${ }^{14}$. C'est le cas encore de certaines théories sur l'identité dans lesquelles on découvre une tension dans le soi selon qu'il se définit par référence à une collectivité ou par rapport à la personne ${ }^{15}$. En dehors de ces réflexions psychosociales, il y a d'autres théories qui ont pour objet la dyna-

Rouergue, [1992] 2004. Alain Touraine, Critique de la modernité, Paris, Fayard, 1992; Alain Touraine, Pourrons-nous vivre ensemble? Égaux et différents, Paris, Fayard, 1997; Alain Touraine, Le Retour de l'acteur, Paris, Fayard, 1984.

12 Alain Ehrenberg, Le Culte de la performance, Paris, Calmann-Lévy, 1991; Alain Ehrenberg, La Fatigue d'être soi. Dépression et société, Paris, Odile Jacob, 1998; Alain Ehrenberg, L'Individu incertain, Paris, Hachette, 1995. Gilles Lipovetsky, L'Ėre du vide. Essais sur l'individualisme contemporain, Paris, Gallimard, 1983.

13 Muriel Darmon, La Socialisation, Paris, Armand Colin, coll. « 128 », 2006.

14 George H. Mead, L'Esprit, le soi et la société, Paris, Presses universitaires de France, [1934] 2006.

15 On lira sur cette thématique le livre dirigé par Catherine Halpern, L'Individu, le groupe, la société, Paris, Éditions Sciences humaines, 2009. 
mique de la socialité et de l'individualité. Il y en a, bien sûr, nombreuses, qui insistent sur l'autonomie des acteurs sociaux; qu'on songe à l'individualisme méthodologique, à l'interactionnisme symbolique ou à l'ethnométhologie. Il y en a d'autres qui mettent l'accent sur la force des structures extérieures : le structuro-fonctionnalisme, le fonctionnalisme, le structuralisme. Et puis il y en a qui conçoivent de manière explicitement dialectique le rapport entre les structures sociales et les actions sociales; la théorie de la structuration d'Anthony Giddens ${ }^{16}$ ou l'analyse complexe d'Edgar Morin ${ }^{17}$ par exemple. Il y a, par ailleurs, plusieurs théories qui évoluent au-delà de ces questionnements : celles, par exemple, de Lev Vygotski ${ }^{18}$, de Norbert Elias ${ }^{19}$ ou de Niklas Luhmann ${ }^{20}$. Mais leur contribution à la polémique de l'homogénéisation et de la différenciation est normalement indirecte. Ces travaux n'ont pas explicitement pour problématique cette polémique même alors que plusieurs d'entre eux produisent des résultats qui devraient sortir le débat de la polarisation et le transporter dans des milieux plus posés. Nous songeons surtout à ces travaux dans lesquels la notion de dialectique est opérationnelle, que ce soit explicitement, comme chez Morin (qui la trouve dans la récursivité et dans la dialogie), ou implicitement, comme chez Mead. Tous ces travaux n'empêchent pas que les deux positions persistent dans une vigoureuse antinomie.

\footnotetext{
16 Anthony Giddens, The Constitution of Society: Outline of the Theory of Structuration, Berkely et Los Angeles, University of California Press, 1984.

17 Edgar Morin, "Complexité : les défis de la méthode ", dans Edgar Morin et Jean-Louis Le Moigne, L'Intelligence de la complexité, Paris, L'Harmattan, 1999, p. 105-169.

18 Lev Vygotski, Pensée et langage, Paris, La Dispute, [1934] 1997.

19 Norbert Elias, La Civilisation des moeurs, Paris, Calmann-Lévy, Pocket, coll. "Agora », [1939] 2002 et La Dynamique de l'Occident, Paris, Calmann-Lévy, Pocket, coll. "Agora ", [1939] 2003.

20 Niklas Luhmann, Systèmes sociaux. Esquisse d'une théorie générale, Québec, Presses de l'Université Laval, coll. «Inter-Sophia », [1984] 2010.
} 


\section{Des travaux empiriques}

La question se pose de savoir laquelle de ces deux analyses est juste. Mais on ne peut répondre à cette question de manière purement spéculative, à moins d'accepter de s'installer dans le champ de la polémique où la vérité est trop souvent celle de la persuasion plutôt que de la démonstration et où les discours tendent à se développer en circuit fermé. Il nous semble donc nécessaire d'opérationnaliser ces positions théoriques et de les soumettre à une observation systématique.

C'est ce que nous avons fait dans Homogénéité et distinction avec notre collègue Ali Reguigui ${ }^{21}$. La recherche était modeste, mais elle avait le mérite d'être empirique et de soumettre les opinions des deux camps à la vérification. Elle avait deux objets, l'un étant les erreurs linguistiques à l'écrit, l'autre, le fait qu'une personne prenne ou non position quand elle s'exprime sur une thématique. Pour chacun de ces deux objets, les indicateurs étaient nombreux. L'échantillon était composé de jeunes de première année universitaire provenant de trois pays ${ }^{22}$ et issus de cinq groupes linguistiques ${ }^{23}$. Pour chacun d'eux, nous recueillions, outre les textes, de nombreuses informations sur la langue de communication, l'exposition aux médias, le statut socioéconomique et sur les représentations. Nos analyses étaient principalement statistiques. Elles ont démontré que les deux positions n'étaient pas mutuellement exclusives. Elles ont signalé des phénomènes d'homogénéisation à l'intérieur des cinq groupes dans le fait, entre autres, que chacun d'eux 1) comportait une probabilité de la fréquence des erreurs linguistiques et 2) présen-

\footnotetext{
21 Simon Laflamme et Ali Reguigui, Homogénéité et distinction, Sudbury, Prise de parole, 2003.

22 Canada, France, Tunisie.

23 1) Des francophones du Canada, qui sont en situation minoritaire dans leur province; 2) des anglophones du Canada, qui sont en situation majoritaire dans leur province; 3) des Français, qui sont dans une situation de quasiunilinguisme institutionnel; 4) des arabisants de Tunisie, qui étudient normalement dans la langue maternelle qui est celle de la majorité; 5) des francisants de Tunisie, qui étudient normalement dans une langue seconde dont le statut symbolique est élevé.
} 
tait une forme d'argumentation dominante. Elles ont aussi identifié des manifestations d'homogénéisation entre les groupes en constatant 1) que la probabilité d'erreur était partout importante, 2) que, dans les sociétés les plus industrialisées, la prise de position était dominante et 3) qu'il y a des formes d'argumentation semblables d'un groupe linguistique à l'autre. Elles ont relevé des signes de différenciation au sein des groupes, entre autres parce que 1) les écarts-types étaient constamment élevés en chacun d'eux, ce qui témoigne de variations interindividuelles, et 2) les façons de manipuler les arguments contraires à la position qui est tenue n'étaient pas toujours les mêmes. Elles ont, enfin, noté qu'il y avait différenciation entre les groupes, notamment en ce que 1) la probabilité d'erreur linguistique n'est pas partout la même, 2) la probabilité de prendre position varie d'un groupe linguistique à l'autre et 3) la forme de l'argumentation varie d'une société à l'autre, même entre des sociétés postindustrielles.

Depuis cette recherche sur les erreurs linguistiques et la production d'opinions, nous n'avons cessé de croiser le dualisme de l'homogénéisation et de la différenciation. Par exemple, nous l'avons trouvé dans une étude sur la possession et l'usage des médias en Ontario selon qu'il s'agit des francophones minoritaires ou des anglophones majoritaires ${ }^{24}$. Nous avons alors observé que les compositions médiatiques des foyers étaient semblables entre les deux groupes linguistiques et que les fréquences d'usage étaient comparables. Les moyennes étaient toujours proches les unes des autres, les faibles inégalités favorisant tantôt un groupe, tantôt l'autre. À la lumière de ces données, on a donc affaire à un fort phénomène d'homogénéisation dans lequel les spécificités de groupes sont minimes. Cependant, dans presque tous les cas, les écarts-types sont importants, ce qui rappelle que s'il y a indifférenciation entre les groupes, il y a malgré tout différenciation dans chacun d'eux. Cette analyse a

24 Simon Laflamme, "Les Médias en Ontario chez les francophones et les anglophones ", Cahiers Charlevoix, Sudbury, Société Charlevoix / Prise de parole, $n^{\circ}$ 6, 2004, p. 251-282. 
par ailleurs montré que les inégalités de moyennes renvoyaient bien plus à l'âge et à l'instruction qu'à la langue ou au statut linguistique. Les jeunes emploient davantage l'informatique que les plus vieux et les plus instruits plus que les autres. Cela vaut pour les deux groupes linguistiques. On a ainsi sous les yeux une manifestation d'homogénéisation dans l'indépendance de l'effet de l'âge et dans celle de l'incidence de l'instruction par rapport au groupe linguistique, mais on a affaire à une hétérogénéisation dans les variations selon l'âge. En outre, comme aucun phénomène ne correspond à des comportements universels, derrière chaque tendance à l'indifférenciation, il y a quelque différenciation.

Nous avons à nouveau rencontré la dualité de l'homogénéisation et de la différenciation dans une analyse qualitative qui portait sur l'usage et la représentation d'Internet chez les jeunes Ontariens selon que leur langue maternelle est le français ou l'anglais ${ }^{25}$. L'analyse a fait état de nombreuses similitudes et de quelques différences. En ce qui a trait à la représentation : Internet était généralement associé à l'information et à la communication, mais jamais par tout le monde dans les groupes; dans les deux groupes, les jeunes pensaient à divertissement quand ils songeaient à Internet, mais cela était plus fréquent chez les anglophones que chez les francophones; il était plus fréquent chez les anglophones que chez les anglophones de signaler les qualités du média, mais dans les deux groupes elles étaient mentionnées. Pour ce qui est de l'usage, les sites qui étaient populaires chez les francophones l'étaient aussi chez les anglophones, mais on notait des variations quant au recours au média relatif à l'instruction et en ce qui a trait à la langue d'utilisation, les francophones exploitaient le médium de façon bilingue, les anglophones tendaient à l'unilinguisme. Il était aisé de constater que le média lui-même uniformisait l'image qu'on pouvait avoir

25 Simon Laflamme, "Usage et représentation d'Internet chez les jeunes. Comparaison entre les francophones et les anglophones du nord de l'Ontario ", Cahiers Charlevoix, Sudbury, Société Charlevoix / Prise de parole, $\mathrm{n}^{\circ}$ 7, 2006, p. 183-228. 
de lui et l'usage qu'on pouvait en faire, mais qu'il n'annihilait pas la différence.

Nous avons aperçu le jeu entre la similitude et la dissimilitude de la socialité dans des travaux, par exemple, sur les aspirations selon l'ethnie, sur la représentation de la santé, sur les catégories socioprofessionnelles, l'identité et l'usage des médias. Chaque fois le travail empirique nous a interdit de négliger l'un ou l'autre des termes de la dualité. Dans l'étude sur les aspirations, nous avons comparé les ambitions juvéniles en matière de scolarisation et de profession selon que les jeunes s'identifient aux francophones, aux anglophones ou aux Premières nations (soit à un peuple amérindien). Les analyses révèlent de grandes similitudes entre les trois populations en ce qu'il est clair que les sociétés postindustrielles avancées diffusent partout des symboliques communes et sollicitent des compétences semblables; mais ces sociétés n’empêchent pas des représentations qui soient spécifiques aux populations ni des variations dans les ensembles d'aspirations qui soient attribuables à l'ethnie ${ }^{26}$. Dans la recherche sur les représentations de la santé, nous avons vérifié si l'auto-évaluation des jeunes de leur santé physique et psychique variait, entre autres, selon le sexe et l'appartenance ethnique. Nous avons découvert que, pour ce qui est de l'état physique, les estimations des filles sont un peu plus critiques que celles des garçons, mais que, dans l'ensemble, les jugements étaient peu enthousiastes. Nous avons noté que le désespoir, la solitude, le découragement, l'emportement, l'ennui étaient communs, mais que ces états d'âme n'étaient pas normalement envahissants. Les moyennes faisaient état de l'étendue des phénomènes, mais les écarts-types mettaient en évidence des variations individuelles. Nous avons remarqué que le sentiment de désespoir différencie les filles des garçons chez les francophones et les anglophones, mais que, chez les jeunes qui s'identifient aux Premières nations, les filles ne connaissent pas davantage cette émotion que les garçons. Ainsi,

$\overline{26}$ Simon Laflamme, «Le Nouveau visage de l'ambition en Ontario français. Le cas du Nord-Est ", Cahiers Charlevoix. Études franco-ontariennes, Ottawa, La Société Charlevoix / Presses de l'Université d'Ottawa, nº 8, 2010, p. 77-110. 
à nouveau, ont voit la postmodernité généraliser des phénomènes, mais sans éliminer les variations individuelles ni les spécificités ethniques ${ }^{27}$. Dans le travail sur les professions, l'identité et l'usage des médias, nous avons montré que les symboliques identitaires avaient peu à voir avec la distribution de la société en professions, qu'elles dépendaient pour beaucoup du rapport que les individus entretiennent avec les médias, de la similitude autant que de la particularité de ces rapports : c'est la complexité des rapports à l'information, par-dessus-tout, qui est responsable de ce que les citoyens ont en partage autant que de ce qui les éloigne ${ }^{28}$.

D'autres travaux empiriques ont fait des constats semblables aux nôtres. Philippe d'Iribarne et ses collaborateurs ont observé que la mondialisation n'anéantissait pas les cultures nationales même au sein des entreprises capitalistes, que si ces entreprises influaient sur les cultures particulières, celles-ci avaient une incidence sur celles-là. Jean-Pierre Poulain a noté des effets évidents d'homogénéisation dans la disparition d'aliments et dans l'expansion de la restauration franchisée, mais il rappelle l'importance des particularismes nationaux et même régionaux ${ }^{29}$. Roger Gervais compare des journaux nationaux et régionaux au Canada et en France et remarque qu'on est loin d'assister à un phénomène d'uniformisation de la presse, que s'il y a des récurrences entre les contenus des journaux nationaux et régionaux, les régionaux n'en ont pas moins une grande autonomie, que s'il y a des ressemblances entre les journaux nationaux de la France et du Canada, les différences sont marquantes ${ }^{30}$.

$27 \quad$ Simon Laflamme, «L'auto-estimation de la santé chez les jeunes du Nord-Est de l'Ontario. Note empirique ", Revue du Nouvel-Ontario (Sudbury), n 32, 2007, p. 101-113.

28 Simon Laflamme, "Catégorie socioprofessionnelle, identité, engagement social et usage des médias. Analyse d'une dynamique complexe ", Revue canadienne de sociologie, vol. 47, $\mathrm{n}^{\circ} 3$, p. 273-292.

29 Jean-Pierre Poulain, Sociologies de l'alimentation, $2^{\mathrm{e}}$ édition, Paris, Presses universitaires de France, [2002] 2011.

30 Roger Gervais, Presse et mondialisation : étude comparée franco-canadienne, op. cit. 


\section{Quelques principes en guise de conclusion}

Ces études rappellent qu'il ne peut pas y avoir une société dans laquelle il y aurait pure homogénéité. Si tous les individus étaient les mêmes, il ne pourrait y avoir d'échange entre eux, car tout serait a priori échangé; il ne pourrait, non plus y avoir de division des tâches et des responsabilités. Si tous les individus étaient différents, ils n'auraient rien en commun, et donc il n'y aurait pas de socialité. Il n'y a jamais eu de société de la "même-itude "; il n'y a jamais eu de société de l'" alter-itude ». Une société ne peut exister que dans la mesure où elle produit aussi bien de la similitude que de la dissimilitude. Une société est par essence le fruit d'une dynamique du semblable et du dissemblable. C'est parce que ses membres ont quelque chose en partage, et donc qu'ils ont quelque ressemblance, qu'ils existent comme collectivité. C'est parce qu'ils ont quelque particularité qu'ils peuvent communiquer entre eux et assurer les diverses tâches qui permettent la reproduction aussi bien de la collectivité que des individus. Et c'est parce que cette dynamique est intrinsèque à l'humaine socialité que cette socialité est historique, que donc la socialité n'est pas ou bien pure reproduction du même, ou bien production aléatoire d'individualités distinctes.

Pourquoi, alors, a-t-on pu croire observer un mouvement simple d'homogénéisation ou de différenciation? La raison en est que la contemporanéité génère de la socialité à une échelle immensément étendue. Des pays comptent des millions de citoyens, parfois sur de vastes territoires. De telles échelles sociétales ne sont possibles que dans la mesure où des symboliques (des héros, des œuvres, des valeurs) peuvent être partagées, où des informations peuvent être transmises virtuellement à tous, où des formations peuvent être suivies en divers lieux, où les individus peuvent communiquer avec leurs proches alors même qu'ils ne sont pas dans un même lieu. Des médias et des institutions y veillent et uniformisent la société. Mais, en même temps, cette étendue sociétale, cette diversité des rapports aux informations et de la relation aux médias génère de la différenciation, de sorte que les individus se développent réellement comme parti- 
cularités. L'erreur, c'est de ne pas voir les transcendances par delà ces particularités ou les spécificités en deçà de ces transcendances. Ce qui est particulier dans la contemporanéité, ce n'est pas la dialectique de l'homogénéisation et de la différenciation, c'est la magnitude de cette dynamique. Une magnitude qui dépasse même la socialité des États-nations, qui fait que des nations différentes ont des valeurs communes en même temps qu'elles reproduisent leur spécificité, en même temps aussi que leurs citoyens respectifs sont à la fois semblables à leurs concitoyens et différents d'eux, pareils et non pareils aux citoyens des autres nations.

Les analyses empiriques ne peuvent pas observer de dynamique, ou de réciprocité, ou de dialectique. Ce sont là des catégories théoriques, avec lesquelles on peut interpréter des données. Elles peuvent constater des corrélations ou des dualismes, ou la coexistence de plusieurs éléments. Dans les travaux que nous avons rappelés, jamais les indicateurs de similitude n’ont écarté ceux de la dissimilitude, jamais les statistiques de la ressemblance n'ont éliminé celles de la ressemblance, jamais les catégories empiriques de l'homogénéité n'ont supprimé celles de la différence. Toujours l'observateur a eu sous les yeux un dualisme. Il n'est pas permis, en science, d'exclure des observations au bénéfice d'une orientation théorique affectionnée; il n'est pas permis, en science, de développer des théories de l'être et de ne pas demander à l'être ce qu'il en pense. Cette constante observation duelle invite à quelque théorisation. Elle incite à s'interroger sur la possibilité d'une société de la "même-itude " et à répondre par la négative. Elle oblige à concevoir une société de l'" alteritude " et à découvrir qu'elle est difficilement imaginable. Que pourrait être une société dans laquelle il n'y aurait aucune différence? Que pourrait être une société qui ne serait que différence? L'histoire humaine n'a donné aucune d'elles à observer. Et si l'on y tient à pousser l'empirie jusqu'à la littérature fantastique, même dans Le Meilleur des mondes d'Aldous Huxley tout le monde n'est pas pareil. Les hypothèses peuvent quand même persister, à des fins théoriques; mais il reste qu'on a sous les yeux, au plan 
empirique, un dualisme, récurrent. Et il nous semble que ce dualisme peut donner lieu à la théorisation d'une double dialectique. Nous croyons, en effet, que, à un type d'homogénéisation correspond un type de différenciation, et que, inversement, chacun de ces types est un processus influant sur l'autre. Corrélativement, on pourrait soulever l'hypothèse que plus est grande l'homogénéisation, plus l'est également la différenciation. A cette dialectique est attachée une structure sociale, plus ou moins démocratique, plus ou moins institutionnalisée, plus ou moins populeuse, plus ou moins segmentée, plus ou moins physiquement étendue, plus ou moins riche. Cette structure sociale, en retour, détermine, elle, la dialectique de l'homogénéisation et de la différenciation.

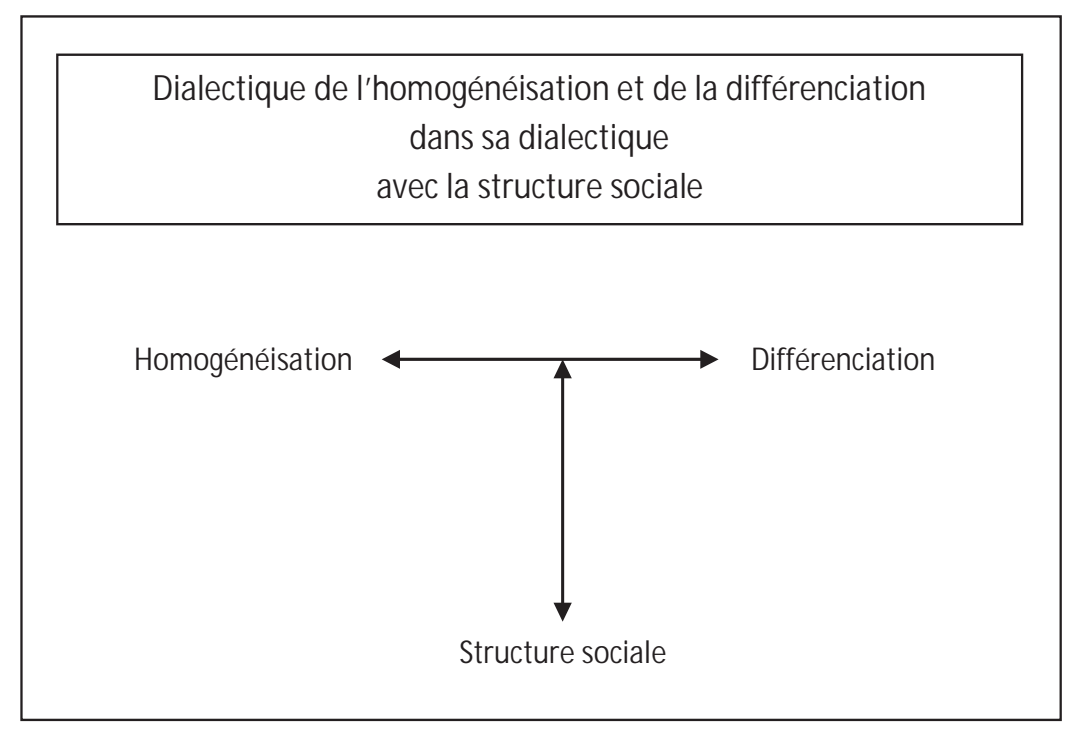




\section{Bibliographie}

Adorno, Theodor W., Modèles critiques, tome 1 [1963], tome 2 [1965], Paris, Payot, 2003.

Baudrillard, Jean, La Société de consommation : ses mythes, ses structures, Paris, Denoël, coll. «Folio Essai », [1970] 1986.

Beck, Ulrich, Pouvoir et contre pouvoir à l'ère de la mondialisation, Paris, Flammarion, [2002] 2003.

Boisvert, Yves, Le Postmodernisme, Montréal, Boréal, 1995.

Bourdieu, Pierre, Sur la télévision. L'emprise du journalisme, Paris, Raison d'agir, 1996.

Darmon, Muriel, La Socialisation, Paris, Armand Colin, coll. « 128 », 2006.

Dubar, Claude, La Crise des identités. L'interprétation d'une mutation, Paris, Presses universitaires de France, 2000.

Durkheim, Émile, De la division du travail social, Paris, Presses universitaires de France, 8 édition, [1893] 1967.

Durkheim, Émile, Les Formes élémentaires de la vie religieuse, Paris, CNRS Éditions, [1912] 2007.

Ehrenberg, Alain, Le Culte de la performance, Paris, Calmann-Lévy, 1991.

Ehrenberg, Alain, La fatigue d'être soi. Dépression et société, Paris, Odile Jacob, 1998.

Ehrenberg, Alain, L'Individu incertain, Paris, Hachette, 1995.

Elias, Norbert, La Civilisation des mours, Paris, Calmann-Lévy, Pocket, coll. "Agora ", [1939] 2002.

Elias, Norbert, La Dynamique de l'Occident, Paris, Calmann-Lévy, Pocket, coll. "Agora ", [1939] 2003.

Gerbner, George, "Mass Media and Human Communication ", dans Frank E. X. Dance (dir.), Human Communication Theory, New York, Holt, Rinehart, 1967, p. 40-57.

Gervais, Roger, Presse et mondialisation : étude comparée franco-canadienne, thèse de doctorat, Université des sciences sociales de Toulouse I, Toulouse, mai 2009.

Giddens, Anthony, Les Conséquences de la modernité, Paris, L'Harmattan, [1991] 2000.

Giddens, Anthony, The Constitution of Society: Outline of the Theory of Structuration, Berkely et Los Angeles, University of California Press, 1984. 
Giddens, Anthony, La Transformation de l'intimité. Sexualité, amour et érotisme dans les sociétés modernes, Rodez, Éditions du Rouergue, [1992] 2004.

Halpern, Catherine, L'Individu, le groupe, la société, Paris, Éditions Sciences humaines, 2009.

Horkheimer, Max, Éclipse de la raison, suivi de Raison et conservation de soi, Paris, Payot, [1949] 1974.

Horkheimer, Max et Theodor W. Adorno, La Dialectique de la raison: fragments philosophiques, Paris, Gallimard, coll. «Tel », [1947] 1983.

Iribarne, Philippe d', La Logique de l'honneur : gestion des entreprises et traditions nationales, Paris, Seuil, 1989.

Iribarne, Philippe d', Alain Henry, Jean-Pierre Segal, Sylvie Chevrier et Tajana Globokar, Cultures et mondialisation. Gérer par-delà les frontières, Paris, Seuil, [1998] 2002.

Klein, Naomi, No Logo. La tyrannie des marques, Arles, Actes Sud, [2000] 2001.

Laflamme, Simon, "L'auto-estimation de la santé chez les jeunes du Nord-Est de l'Ontario. Note empirique ", Revue du Nouvel-Ontario (Sudbury), no 32, 2007, p. 101-113.

Laflamme, Simon, "Catégorie socioprofessionnelle, identité, engagement social et usage des médias. Analyse d'une dynamique complexe ", Revue canadienne de sociologie, vol. 47, n 3, p. 273-292.

Laflamme, Simon, "Les Médias en Ontario chez les francophones et les anglophones ", Cahiers Charlevoix, Sudbury, Société Charlevoix / Prise de parole, $n^{\circ} 6,2004$, p. 251-282.

Laflamme, Simon, «Le Nouveau visage de l'ambition en Ontario français. Le cas du Nord-Est ", Cahiers Charlevoix. Études franco-ontariennes, Ottawa, La Société Charlevoix / Presses de l'Université d'Ottawa, $\mathrm{n}^{\circ}$ 8, 2010, p. 77-110.

Laflamme, Simon, "Usage et représentation d'Internet chez les jeunes. Comparaison entre les francophones et les anglophones du nord de l'Ontario ", ", Cahiers Charlevoix, Sudbury, Société Charlevoix / Prise de parole, $\mathrm{n}^{\circ}$ 7, 2006, p. 183-228.

Laflamme, Simon et Ali Reguigui, Homogénéité et distinction, Sudbury, Prise de parole, 2003.

Lerner, Daniel, The Passing of Traditional Society, New York, Free Press, 1958.

Lipovetsky, Gilles, L'Ère du vide. Essais sur l'individualisme contemporain, Paris, Gallimard, 1983. 
Luhmann, Niklas, Systèmes sociaux. Esquisse d'une théorie générale, Québec, Presses de l'Université Laval, coll. "Inter-Sophia », [1984] 2010.

Marx, Karl, Le Capital, tome 1, Presses universitaires de France, [1867] 1970.

Marx, Karl, Introduction à la critique de l'économie politique, Montreuil, Altiplano, [1857] 2008.

Marx, Karl et Fredrich Engels, Manifeste du parti communiste, Anjou (Québec), Éditions CEC, [1847] 2009.

Mattelart, Armand, Multinationales et système de communication, Paris, Anthropos, 1976.

Mead, George H., L'Esprit, le soi et la société, Paris, Presses universitaires de France, [1934] 2006.

Miège, Bernard, La Société conquise par la communication, Grenoble, Presses de l'Université de Grenoble, coll. "Logiques sociales ", 1996.

Morin, Edgar, "Complexité : les défis de la méthode », dans Edgar Morin et Jean-Louis Le Moigne, L'Intelligence de la complexité, Paris, L'Harmattan, 1999, p. 105-169.

Poulain, Jean-Pierre, Sociologies de l'alimentation, $2^{\mathrm{e}}$ édition, Paris, Presses universitaires de France, [2002] 2011.

Touraine, Alain, Critique de la modernité, Paris, Fayard, 1992.

Touraine, Alain, Pourrons-nous vivre ensemble? Égaux et différents, Paris, Fayard, 1997.

Touraine, Alain, Le Retour de l'acteur, Paris, Fayard, 1984.

Weber, Max, Économie et société, tome 1, Paris, Plon, [1921 (posthume)] 1971.

Weber, Max, Économie et société dans l'antiquité, Paris, La Découverte, [1909] 1998.

Weber, Max, Hindouisme et bouddhisme, Paris, Flammarion, [1916] 2003. 